\title{
Role of Hysteroscopy in the Diagnosis of Abnormal Uterine Bleeding In Perimenopausal Age Group
}

\author{
Dr. K. Padmavathi*, Dr. K. Kurmanadh**, Dr.K.V.S.Deepak*** \\ Dept.ofobs\& gynaecology, Andhra medical college, Visakhapatnam. \\ *assist. Prof of gynaecology, **associate. Prof of anaesthesiology, ${ }^{* * *}$ jr.resident
}

\section{Introduction}

Menstrual dysfunction is the cause of discomfort, inconvenience, and disruption to healthy lifestyle which affects millions of women in both developed and developing world. It is one of the most common presenting symptoms in the gynaecological clinic. Approximately $20 \%$ of patients presenting to a gynaecologist have this complaint.

Goals of clinical management are primarily dependent upon attaining a correct etiological diagnosis. The history, physical and pelvic examination attempt to determine the site of the bleeding and its source. Information gathered from this will suggest which direction the investigation would take. Traditionally Dilatation and Curettage and Ultrasonography were the most common investigations employed in the evaluation of the causes of abnormal uterine bleeding.

Dilatation and Curettage is a blind procedure and the endometrium has to be sent to the pathologist to study histological patterns and for the report. Ultrasonography clearly depicts the uterine contour and the status of uterine the ovary, but fails to provide adequate information regarding the endometrium.Hysteroscopy has ushered a new era in the evaluation of abnormal uterine bleeding. By direct visualisation of the cavity it is able to pin point the aetiology in majority of cases. It can accurately detect endometrial hyperplasia and aids in the early diagnosis of endometrial carcinoma and uterine polyps.

D\&C has long been the diagnostic gold standard for abnormal uterine bleeding. However, only 70-80\% of the endometrium can be curetted. Polyps and sub mucous fibroids are frequently undetected by curettage alone. The judicious use of hysteroscopy to manage this medical entity adds a new dimension in handling this often perplexing problem. This study has been taken up to analyse the place of hysteroscopy in the evaluation of Abnormal Uterine Bleeding in terms of accuracy of hysteroscopic findings and the contribution of the procedure to clinical diagnosis. It also aims to correlate hysteroscopic findings with histopathological results.

\section{Aims And Objectives}

The aim of the study is to evaluate the role of hysteroscopy in the diagnosis of cases with abnormal uterine bleeding in the peri menopausal age groupand its correlation with histopathological findings.

\section{HISTORICAL REVIEW:}

\section{Review Of Literature}

"A Vigilant eye in the uterine cavity is better than numerous blind curettages", quoted Lindmann, about future of hysteroscopy. In 1907, David demonstrated the first contact hysteroscopy, which was useful for the diagnosis of uterine disorders. In 1914, Heinberg devised a system for irrigating the uterine cavity to rinse off the blood that often covered the lens and hindered the lesion. In 1925, Rubin insufflated the uterine cavity with $\mathrm{CO}_{2}$ instead of water. In 1927, Mikulicz-Radecki and Freund collaborated to produce a 'curettoscsope' with biopsy taking capability and corneal electro coagulation.

Valle in 1981 studied 419 pre-menopausal and 134 post-menopausal patients who had abnormal uterine bleeding. Hysteroscopy was performed prior to D\&C. In 352 patients an abnormality on hysteroscopy was detected, such as endometrial polyps, sub mucous leiomyomas, and intra-uterine adhesions. He concluded that hysteroscopy provides a precise and accurate adjunct to traditional methods of diagnosing intra-uterine abnormalities, particularly, focal lesions missed at curettage.

Dewits and Vleugels in 2003 evaluated 1045 hysteroscopies performed over 6 years retrospectively. Normal cavity was found in $54.2 \%$. Most common abnormal findings were fibroids(21\%) and endometrial polyps(14.4\%). Hysteroscopically diagnosed hyperplasia of the endometrium was confirmed in only less than half the cases. Endometrial carcinoma was suspected on hysteroscopy in 2 cases of a total of 7 proven cases. Diagnostic hysteroscopy is a valuable tool in diagnosing structural intra-cavital pathology, very suitable for outpatient clinic. 


\section{HYSTEROCOPY}

Hysteroscopy is a technique in which an endoscope is introduced in the cervical canal and uterine cavity through vagina for visualization of endocervical canal and uterine cavity and fibro optical transmitted light provide illumination, distention of uterine cavity is obtained with appropriate media and video camera attached to the telescope allows monitoring on a television screen with magnification.

\section{CONTRAINDICATIONS FOR HYSTEROSCOPY}

\section{ABSOLUTE:}

1. Recent or existing uterine infection:

2. Pregnancys

3. Profuse uterine bleeding: In patients with excessive uterine bleeding hysteroscopy cannot be performed satisfactorily regardless of the distension medium used.

4. Cervical malignancy: Because of the possibility of spreading the disease due to cervical manipulation, patients with known carcinoma cervix should be excluded.

\section{RELATIVE:}

1. Adenocarcinoma of endometrium, when the operator is not familiar with this disease

2. Marked cervical stenosis

3. Operator unfamiliarity with instrumentation and technique.

\section{COMPLICATIONS, PREVENTION AND MANAGEMENT}

When the appropriate technique and selection of patients are observed, hysteroscopy has practically has no complications. However because some blind manipulations are required in sounding the uterine activity or dilatating the endocervical canal, uterine perforation may occur.

Uterine perforation, Infection, Bleeding, Medium related complication, Complications related to intra-uterine operations

\section{Source of Data:}

\section{Materials And Methods}

The present study "THE ROLE OF HYSTEROSCOPY IN ABNORMAL UTERINE BLEEDING IN PERI MENOPAUSAL AGE GROUP AND ITS HISTOPATHOLOGICAL CORRELATION" is a prospective study, which has been carried out in the Department of Obstetrics and Gynaecology, King George Hospital, Visakhapatnam.

The material for the present study was collected from patients who attended and admitted in the Department of Obstetrics and Gynaecology with Abnormal Uterine bleeding. Fifty cases of AUB were taken up for the study. All the patients in this study underwent Hysteroscopy followed by Dilatation and Curettage and the curetting were sent for Histopathology analysis.

The period of study was from January 2010 to June 2011.The results of Hysteroscopy and Endometrial Histopathology were studied and analysed. The analysed data was compared with other series in literature and discussed. sAll patients were well informed about the study in all aspects.

\section{Inclusion Criteria:}

1. Patients with age between 37-50 years with abnormal uterine bleeding.

2. Both parous and nulliparous women

3. Patients who do not require any emergency management

\section{Exclusion Criteria:}

1. Cases with large or multiple fibroids

2. Infection in the uterine tract

3. Cases of carcinoma cervix

Cases were selected by diagnosis on History, General physical examination, Abdomen and Pelvic Examination and Routine investigations. Patients were advised nil by month on the day of Hysteroscopy. They were prepared as for any other surgical procedure.

Laboratory investigations: complete blood counts, urine examination, HIV, HBsAg, Blood grouping and typing, CT, BT, Blood urea, Serum creatinine, Blood glucose, ECG, Thyroid profile and ultrasonography.

\section{INTERVENTIONS INCLUDED:}

1.Anaeshesia:In this study, Hysteroscopy was performed under IV anaesthesia

Drugs used: Diazepam 2mg, Atropin $0.6 \mathrm{mg}$ and pentazocine 
2.instruments and materials required:

Hysteroscope, Distension medium, Sponge holding forceps, Sims speculum, Cervical tenaculum, Cervical dilators,Uterine sound

\section{Procedure}

Patient should be in fasting for about 6 hours before the procedure and anaesthesia given and tetanus toxoid given to prevent tetanus. After keeping the patient in dorsal lithotomy position, vulvar area and vagina are cleansed with antiseptic solution and draped.Per vaginal examination is done to note the direction of the cervix and position of the uterus and speculum is introduced. Cervix is held with vulsellum and cervix is dilated using cervical dilators and telescope is introduced through the external os after fitting it with light source and distension medium using normal saline. By gentle manipulation telescope is introduced slowly.

Cervical canal is visualised in its totality and once the junction between the cervix and uterus is passed, the uterine activity is observed. Once the uterine cavity is entered, the telescope is positioned at the centre with the distal tip being positioned at a distance of $1.5-2 \mathrm{~cm}$ from the fundus while holding the light cable and fixing the camera, the telescope is rotated through 180 degree clockwise and 180 degree anti clockwise. Due to the 30 degree angle of vision, the uterine cavity can be observed.

The uterine cavity should be explored in a systematic fashion after looking in the endocervical canal, right lateral wall, right corneal recess, tubal ostium, fundus left ostium, left corneal recess, left lateral wall, anterior wall and then posterior wall. Biopsy of the suspicious lesions and from areas of hyperplasia was taken and endometrial polyps were removed and sent for histopathological examination.

\section{Observation And Results}

In the present study, Panoramic hysteroscopy was performed using 4mm Hysteroscope with 30 degrees s oblique in 50 patients presented with Abnormal Uterine Bleeding followed by Dilatation and Curettage. The curetted endometrium was sent for histopathological analysis.

Table-1: Distribution of cases according to age group

\begin{tabular}{|l|l|l|l|}
\hline Age Group(years) & No of patients & Abnormal hysteroscopic findings & Percentage \\
\hline $37-40$ & 8 & 6 & 75 \\
\hline $41-45$ & 30 & 26 & 86.67 \\
\hline $46-50$ & 12 & 10 & 83.33 \\
\hline
\end{tabular}

Out of the 50 patients, 30 were from the age group $41-45$ years

$86.67 \%$ of them showed abnormal hysteroscopic findings

$83.33 \%$ of those in the age group $46-50$ years showed abnormal hysteroscopic findings

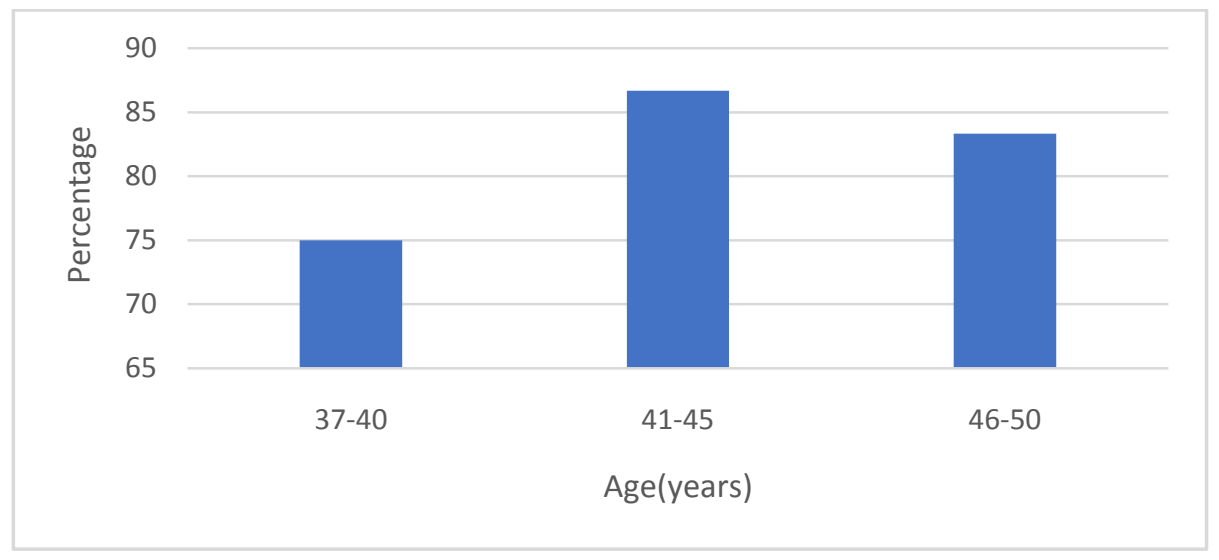

Table II: Distribution of cases according to the duration of symptoms

\begin{tabular}{|l|l|l|l|}
\hline Duration & No of patients & Abnormal hysteroscopic findings & Percentage \\
\hline$<6$ months & 10 & 7 & 70 \\
\hline 6 months- 1 year & 12 & 10 & 83.33 \\
\hline$>1$ year & 28 & 26 & 92.86 \\
\hline
\end{tabular}

Only $70 \%$ of those who had symptoms for less than 6 months and Abnormal hysteroscopic findings as opposed to $92.86 \%$ of those who had symptoms for greater than 1 year. This indicates that longer the duration of symptoms greater are the chances of having Abnormal hysteroscopic findings 
Role Of Hysteroscopy In The Diagnosis Of Abnormal Uterine Bleeding In Perimenopausal Age ..

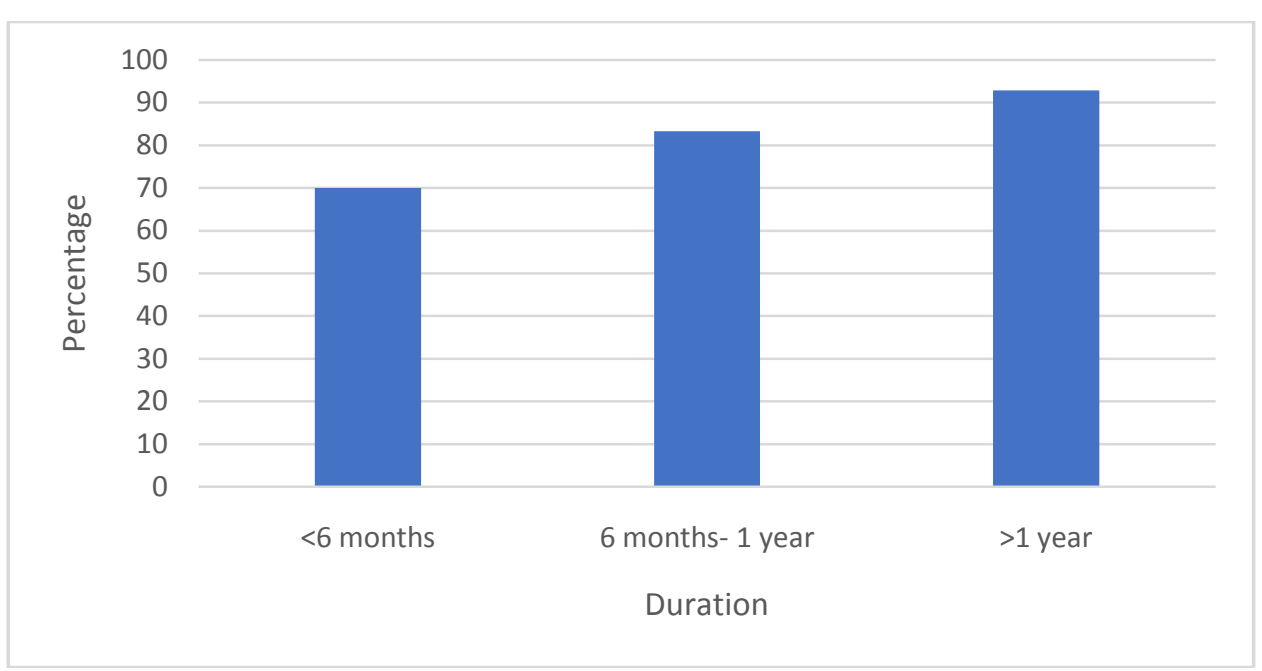

Table III: Duration of cases according to socio economic status

\begin{tabular}{|l|l|l|l|}
\hline Socio economic status & No of patients & Abnormal hysteroscopic findings & Percentage \\
\hline Low & 40 & 34 & 85 \\
\hline Middle & 10 & 8 & 80 \\
\hline High & - & - & - \\
\hline
\end{tabular}

When the results were analysed in relation to socio economic status, abnormal hysteroscopic findings were seen in $85 \%$ of those from Low socio economic status and in $80 \%$ of Middle class women.

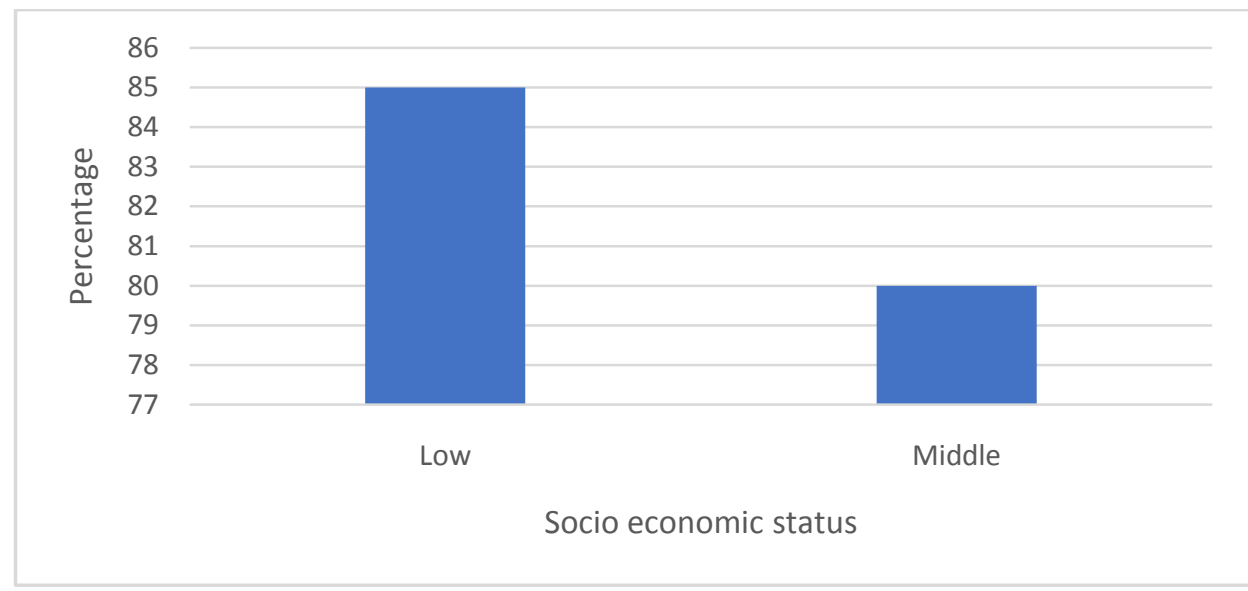

Table IV: Distribution of cases in association with Medical disorders

\begin{tabular}{|l|l|l|l|}
\hline Medical Disorder & No of patients & Abnormal hysteroscopic findings & Percentage \\
\hline Diabetes Mellitus & 6 & 6 & 100 \\
\hline Hypertension & 12 & 13 & 83.33 \\
\hline Thyroid Disorder & 2 & 2 & 100 \\
\hline
\end{tabular}

When the results were analysed in terms of medical disorders. Out of 50 patients, 6 had Diabetes Mellitus and all of them showed Abnormal hysteroscopic findings. Out of the 12 hypertensive patients, $83.33 \%$ showed abnormal findings. This indicates that Diabetes Mellitus is a significant risk factor.

Table V: Distribution of cases in association with Parity

\begin{tabular}{|l|l|l|l|}
\hline Parity & No of Patients & Abnormal Hysteroscopic findings & Percentage \\
\hline Nullipara & 1 & 1 & 100 \\
\hline Primipara & 4 & 4 & 100 \\
\hline Multipara & 32 & 26 & 81.25 \\
\hline Grand multipara & 13 & 11 & 84.61 \\
\hline
\end{tabular}

In this study, one Nulliparous and 4 Primiparous patients were included and all of them showed Abnormal Hysteroscopic findings.82.93\% of women with more than 2 children showed Abnormal Hysteroscopic findings. 
Table VI: AUB and Hysteroscopic findings

\begin{tabular}{|l|l|l|}
\hline Hysteroscopic finding & No of Patients & Percentage \\
\hline Normal & 8 & 16 \\
\hline Endometrial Hyperplasia & 21 & 42 \\
\hline Endometrial Polyps & 12 & 24 \\
\hline Atrophic Endometrium & 4 & 8 \\
\hline Fibroid Uterus & 4 & 8 \\
\hline Malignancy & 1 & 2 \\
\hline
\end{tabular}

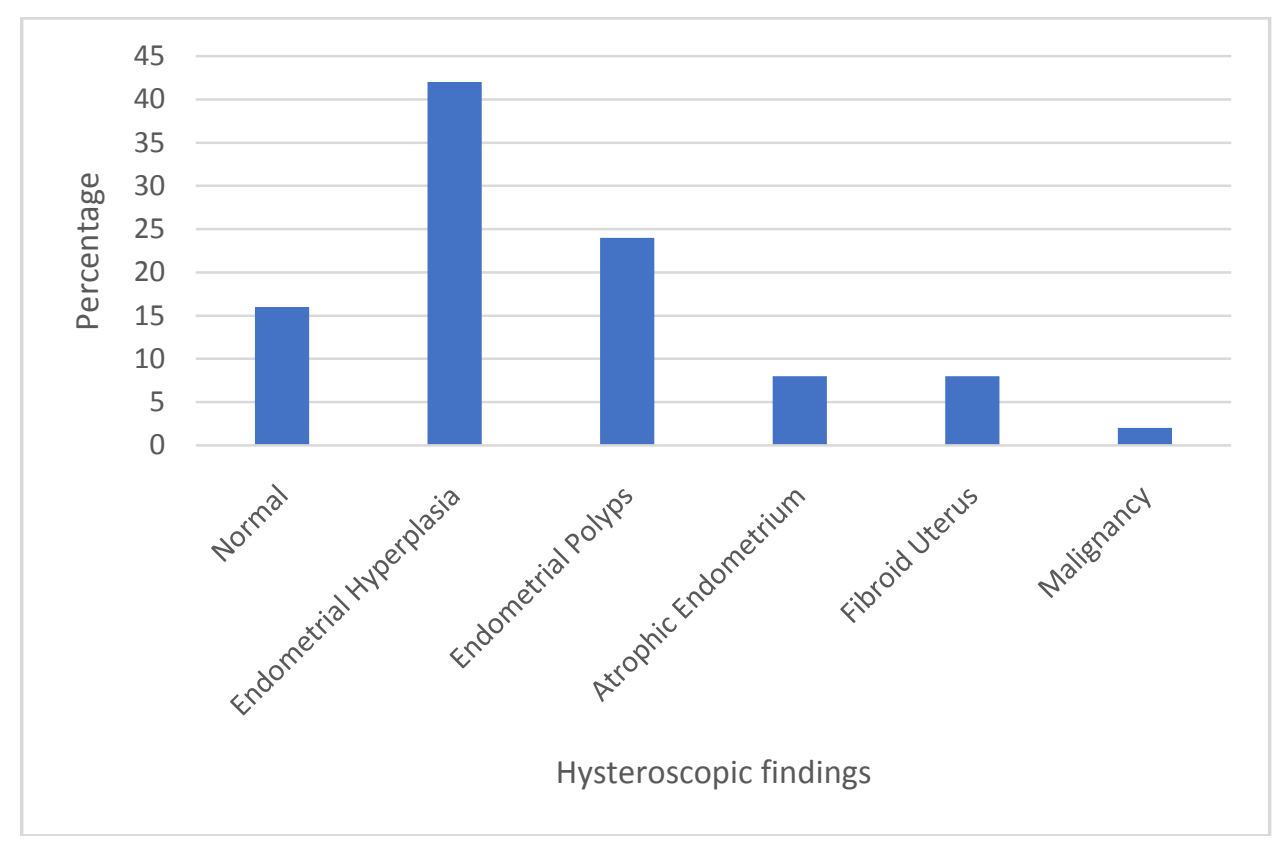

Abnormal findings were seen in 42 patients (84\%), while in the remaining 8 patients(16\%), no abnormality was detected(negative hysteroscopic view). The most common abnormality was endometrial hyperplasia seen in $42 \%$ of patients followed by endometrial polyps seen in $24 \%$ of the patients.
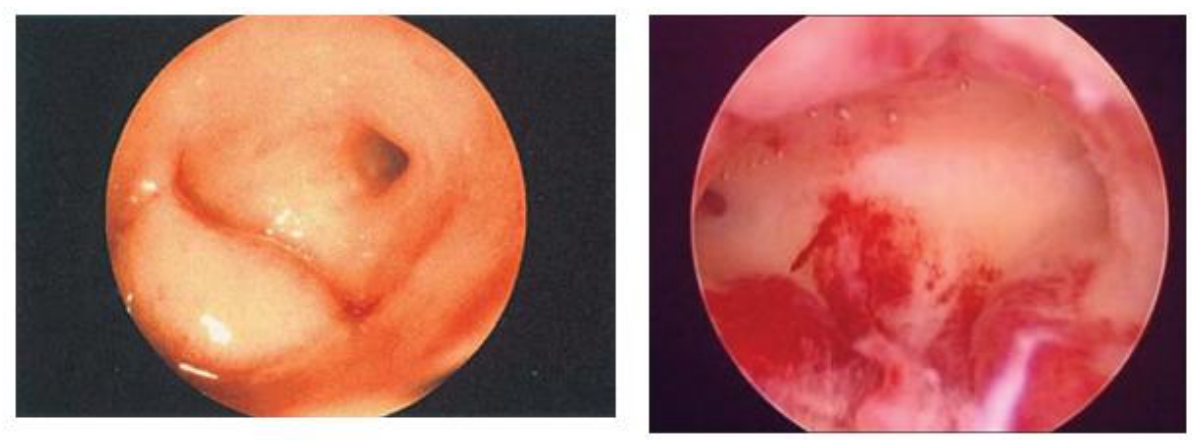

Table VII: Ultrasound findings and its correlation with hysteroscopic findings

\begin{tabular}{|l|l|l|}
\hline Ultrasound findings & Hysteroscopic Findings & No of Patients \\
\hline Endometrial thickness $<8 \mathrm{~mm}$ & Normal & 3 \\
& Endometrial polyp & 8 \\
& Atrophic endometrium & 4 \\
\hline Endometrial thickness $>8 \mathrm{~mm}$ & Normal & 3 \\
& Endometrial polyp & 4 \\
& Hyperplasia & 21 \\
& Fibroid & 4 \\
\hline Echogenic mass & Proliferative growth & 1 \\
\hline
\end{tabular}

Endometrial thickness was measured in all the patients ultrasonographically. 17 patients had endometrial thickness less than $8 \mathrm{~mm}$. Of them 5 showed normal Hysteroscopic findings and 12 showed abnormal hysteroscopic findings(70.59\%). Of the patients who had endometrial thickness more than $8 \mathrm{~mm}$, $29(90.62 \%)$ showed abnormal hysteroscopic findings of which 21 had endometrial hyperplasia. 1 patient showed an echogenic mass which turned to have a proliferative growth. 
Role Of Hysteroscopy In The Diagnosis Of Abnormal Uterine Bleeding In Perimenopausal Age ..

Table VIII: Abnormal Uterine bleeding and histopathologic reports

\begin{tabular}{|l|l|l|}
\hline Findings & HPE Report & Percentage \\
\hline Normal & 15 & 30 \\
\hline Endometrial hyperplasia & 22 & 44 \\
\hline Endometrial Polyps & 7 & 14 \\
\hline Atrophic endometrium & 5 & 10 \\
\hline Fibroids & - & - \\
\hline Malignancy & 1 & 2 \\
\hline
\end{tabular}

Of the 50 cases, $44 \%$ showed endometrial hyperplasia followed by endometrial polyps in $14 \%$. $30 \%$ of the patients showed normal histopathological findings.
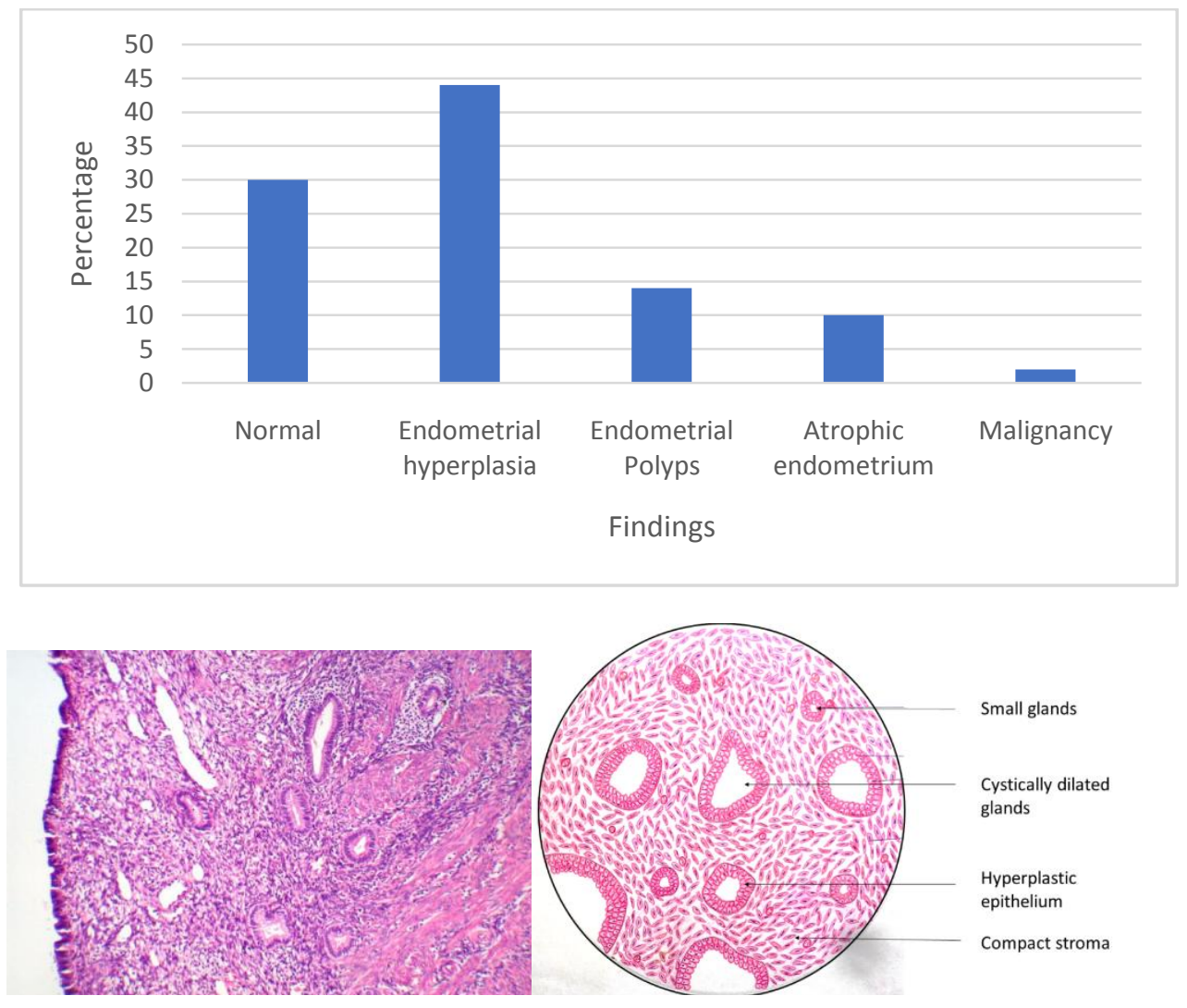

Table IX: Correlation of hysteroscopic findings with histopathological reports

\begin{tabular}{|l|l|l|}
\hline Findings & Hysteroscopic findings & Histopathological findings \\
\hline Normal & 8 & 15 \\
\hline Endometrial hyperplasia & 21 & 22 \\
\hline Endometrial Polyps & 12 & 7 \\
\hline Atrophic endometrium & 4 & 5 \\
\hline Sub mucous Myoma & 4 & - \\
\hline Malignancy & 1 & 1 \\
\hline
\end{tabular}

Of the 50 cases, $16 \%$ cases were normal on Hysteroscopy whereas $30 \%$ showed normal findings on histopathology. $24 \%$ of cases were shown to have endometrial polyps on hysteroscopy whereas only $14 \%$ of endometrial polyps were found on histopathology. 2 false negative findings were observed with hysteroscopy, 1 case of endometrial atrophy and the other a case of endometrial hyperplasia. 1 case which showed endometrial hypertrophy on hysteroscopy was to be normal on histopathology.

\section{Discussion}

There are several studies evaluating the efficacy of different diagnostic methods in the assessment of intracavitary and endometrial lesions. It is accepted that transvaginal sonography has a good sensitivity (Alcazar et al. 1996, Mencaglia 1995) can be used as a first step in the evaluation of a women with post-menopausal bleeding, but hysteroscopy is more specific. The combined use of hysteroscopy and biopsy leads to near $100 \%$ accuracy in the diagnosis of endometrial neoplasia and its precursors (Mencalcial 1995). Endometrial thickness of more than $8 \mathrm{~mm}$ in perimenopausal women is taken as abnormal and warrants further testing but it cannot 
differentiate accurately between hyperplasia, polyps and endometrial carcinoma(Conoscenti et al 1995, Maia et al 1996). In our series, 32 patients had endometrial thickness $>8 \mathrm{~mm}$. Ofthem 21 showed endometrial hyperplasia, 4 had endometrial polyps, 4 had fibroids and only 5 cases showed normal findings. One case which showed echogenic mass on ultrasonography showed proliferative growth.

\section{Normal and Abnormal findings at hysteroscopy in various series:}

\begin{tabular}{|l|l|l|l|l|}
\hline SI No & Author(year) & No of Cases & Normal findings & Abnormal findings \\
\hline 1 & Wamsteker(1984) & 199 & $60 \%$ & $58.5 \%$ \\
\hline 2 & Loffer(1989) & 91 & $48.66 \%$ & $51.44 \%$ \\
\hline 3 & Sheth(1990) & 51 & $44 \%$ & $56 \%$ \\
\hline 5 & Paransis(1992) & 96 & $73.95 \%$ & $26.05 \%$ \\
\hline 6 & Numann(1999) & 85 & $55.2 \%$ & $44.8 \%$ \\
\hline 7 & Panda(1999) & 66 & $46.6 \%$ & $53.4 \%$ \\
\hline 8 & Trotsenburg(2000) & 819 & $66 \%$ & $34 \%$ \\
\hline 9 & Garuti(2001) & 1500 & $61.8 \%$ & $38.2 \%$ \\
\hline 10 & De Wit AC(2003) & 1045 & $54.2 \%$ & $45 \%$ \\
\hline 11 & Present series & 50 & $16 \%$ & $84 \%$ \\
\hline
\end{tabular}

Of the 42 cases with abnormal findings on hysteroscopy, commonest seen was Endometrial hyperplasia(21 cases, $42 \%$ ), followed by endometrial polyps(12 cases, $24 \%$ ) and sub mucous myoma(4 cases, $8 \%$ ). Panda found endometrial hyperplasia in $28.3 \%$, Wamstaker found endometrial polyp in $19 \%$, endometrial hyperplasia in $12.2 \%$ and sub mucous myoma in $7.8 \%$, Trotsenburg observed myomas and polyps in $14 \%$ and de Lewit reported myomas in $21 \%$ and polyps in $14.4 \%$. Hysteroscopy diagnosed all cases of endometrial polyps and sub mucous myomas, and almost all cases of endometrial hyperplasia. Sheth reported $81.8 \%$ accuracy in diagnosis of polyps and myomas, while Garuti reported $95.4 \%$ specificity in the diagnosis of polyps. In the present study, hysteroscopy made two false negative diagnosis and showed 1 case of endometrial hyperplasia and 1 case of endometrial atrophy as normal. It also diagnosed 1 case to be normal but I showed endometrial hyperplasia on histopathology.

\section{Summary And Conclusion}

Abnormal uterine bleeding in the perimenopausal women is much more sinister than in the reproductive age group and warrants thorough investigation, as $84 \%$ of them had detectable endometrial abnormality.The main aim of evaluation of abnormal uterine bleeding in this age group patients is to rule out endometrial hyperplasia and endometrial polyps which may transform into malignancy if undetected and untreated.Patients with risk factors like diabetes mellitus, hypertension, obesity etc., should be evaluated as $100 \%$ of those who are obese and diabetic and $83.33 \%$ of those who are hypertensive were shown to have abnormal findings in our series.Patients with abnormal uterine bleeding must be subjected to one of the three diagnostic modalities i.e, transvaginal ultrasonography, Hysteroscopy or dilatation and curettage.Hysteroscopy was found to be more accurate in detecting endometrial hyperplasia and endometrial polyps Endometritis is one condition which requires histopathological documentation and is not detected by hysteroscopy or ultrasonography.

Hysteroscopy allows direct visual inspection of the uterine cavity and endocervical canal and can help to ensure that any foci of abnormal appearing endometrium are not missed. Also biopsy can be taken from the site for histopathological analysis.Even though transvaginal sonography has a good sensitivity, it fails to differentiate between endometrial polyps, hyperplasia and endometrial carcinoma.Hysteroscopy is more specific as a diagnostic tool in these cases and the combined use of hysteroscopy and biopsy leads to near $100 \%$ accuracy.

An additional advantage with hysteroscopy is that the diagnostic procedure can be extended into an operative procedure to tackle benign conditions like polyps and sub mucous fibroids.Hysteroscopy is safe, reliable and quick procedure in the diagnosis of cases with abnormal uterine bleeding.Hysteroscopy can supplement and enhance the accuracy of tissue diagnosis, so hysteroscopically directed biopsy would be an ideal procedure in cases of abnormal uterine bleeding where facilities are available.If the abnormal uterine bleeding is evaluated by hysteroscopy and it reveals no signs of abnormality, no further investigation of these patients is necessary. Thus by doing investigations like hysteroscopy in women with abnormal uterine bleeding, justice to the patient can be done in the diagnosis and management.

Hysteroscopy is now being done as an office procedure, hospital admission and anaesthesia are avoided. 
[1]. Baggish M.S Obstetrics and Gynaecology, 54:350,1979

[2]. Christine Bain, David E.Parkin, Kevin G.Cooper - British Journal of Obstetrics and Gynaecology July,2002. Is outpatient hysteroscopy more useful than endometrial biopsy alone for the investigation of abnormal uterine bleeding in unselected premenopausal women.

[3]. De Wit AC, Vleugels MP, De Kruif JH, Diagnostic hysteroscopy, valuable diagnostic tool in the diagnosis of structural intra cavitary pathology and endometrial hyperplasia or carcinoma. European journal of Obstetrics and Gynaecology Spetember,2003.

[4]. Dysfunctional uterine bleeding D.A Davey

[5]. Jacques Barbot - Hysteroscopy for abnormal uterine bleeding

[6]. Jeffocoate's principles of gynaecology $5^{\text {th }}$ edition

[7]. Kapur Krishan, Biswas Manash: Journal of Obstetrics and Gynaecology. April 2005

[8]. Linda D. Bradley, MD - Abnormal uterine bleeding

[9]. Loffer FD. Obstetrics and gynaecology,1986

[10]. Maia H Jr. Barabosa IC Farias JP. Ladipo Oa. CoutinnoEm\& M Interational journal of obstetrics and gynaecology.

[11]. Managing abnormal uterine bleeding in peri menopausal women. Pedro A. Poma,MD

[12]. Manual of hysteroscopy- diagnosis and surgery Luca Mencaglia MD and Jacques E.Hamou,MD

[13]. Mencaglia - Obstetrics and Gynaecology clinics North America, September, 1995

[14]. Michael S. Baggish - Distending media for panoramic hysteroscopy.

[15]. Novak ER and Woodruff JD. Novak's gynaecological and obstetric pathology with clinical and endocrine relations $8^{\text {th }}$ edition

[16]. Pedro A. Poma MD - Managing abnormal uterine bleeding in peri menopausal women.

[17]. Progress in obstetrics and gynaecology volume 13. By John Studd

[18]. Rafael F Valle - office hysteroscopy

[19]. Recent advances in Obstetrics and Gynaecology by John Bonnar, Volume 22

[20]. Role of transvaginal ultrasonography, diagnostic hysteroscopy and dilatation and curettage in cases of menorrhagia in peri menopausal age group - Journal of Obstetrics and Gynaecology , 01 September 2002.

[21]. Shaw's textbook of Obstetrics and Gynaecology, $14^{\text {th }}$ edition

[22]. Walton SM. Macphail S. The value of hysteroscopy in post-menopausal and peri-menopausal bleeding, Journal of Obstetrics and Gynaecology. 